\title{
Analytical SIR for Cross Layer Channel Model
}

\author{
Abdurazak Mudesir \\ Jacobs University Bremen, School of Engineering and Science, Research 1, \\ Campus Ring 12. 28759 Bremen \\ Germany \\ Harald Haas \\ The University of Edinburgh, Institute for Digital Communications, \\ The Kings Buildings Edingburgh EH9 3JL \\ UK
}

\section{Introduction}

In a wireless communication environment characterized by dynamically-varying channels, high influence of interference, bandwidth shortage and strong demand for quality of service (QoS) support, the challenge for achieving optimum spectral efficiency and high data rate is unprecedented. One of the bottlenecks in achieving these goals is modeling of the propagation environments Yun \& Iskander (2004).

The commonly used radio propagation models only account for the large scale path loss Rappaport (2001) or only multipath propagations Alouini \& Goldsmith (1997), which are incomplete for studying realistic system deployment scenarios. The authors in Alouini \& Goldsmith (1997) calculate the capacity of Nakagami multipath fading (NMF) channels assuming that the carrier-to-noise ratio (CNR) is gamma distributed. This assumption neglects the effects of shadowing and large scale path loss. This chapter presents an "exact" pdf derived from a model which is more closely related to a realistic deployment scenario. With the results provided here, it is possible to calculate more precise capacity figures. Moreover since the new path loss model takes into consideration the interaction of the system or data link layer with the physical layer (PHY), it is particularly important in studying the cross-layer interaction between MAC and PHY layer.

The general approach of cross-layer design is to maintain the already established layered architecture, capture the important information that influences other layers, exchange the information between layers and implement adaptive protocols and algorithms at each layer to optimize the system performance Xia \& Liang (2005).

Adhering to the idea of cross-layer solutions, many researchers are working towards finding viable cross layer designs spanning the different layers of the OSI model. Yeh and Cohen proposed a generalized analytical framework for cross-layer design focusing on resource allocation in the presence of multi-access fading channels Yeh \& Cohen (2003). Ahn Ahn et al. (2002) uses the information from the medium access control (MAC) layer to do rate control at the network layer.

Cross-layer design relies on channel quality information. The channel quality can be captured by a single parameter, namely the received SIR. The SIR between two communicating nodes 
will typically decrease as the distance between the nodes increases, and will also depend on the signal propagation and interference environment. Moreover, the SIR varies randomly over time due to the propagation environment and interference characteristics. Therefore modeling the SIR on the assumption of the cellular structure and the well known path loss model that ignores the small scale fading would not be applicable to self-configuring cross-layer design. Therefore analytical derivation of the pdf of SIR is a crucial step in constructing efficient crosslayer design.

Tellambura in Tellambura (1999) uses a characteristic function method to calculate the probability that the SIR drops below some predefined threshold (probability of outage) under the assumption of Nakagami fading. Zhan Zhang (1996) also uses a similar characteristic function approach to derive outage probability for multiple interference scenario. These papers give a significant advantage in reducing the computational complexity involved in solving multiple integrals in SIR computation. But, a major shortcoming of these and other similar papers Zorzi (1997) is that, only the small scale fading (physical layer) or large scale fading (data link layer) is considered in analytically deriving the SIR statistics.

The rest of this chapter is organized as follows. In Section II the system model considered is presented and in Section III the analytical derivation is described in detail. Section IV provides the numerical and the simulation results. Section V concludes the chapter.

\section{System Model and Problem Formulation}

For simplicity the cell layout used to derive the pdf of the SIR assumes circular cells, as shown in Fig. 1, with maximum cell radius $R_{\mathrm{c}}$ instead of hexagonal cells. The cells are randomly positioned resulting in potentially overlapping cells. Randomly positioned cells model an important network scenario, which lacks any frequency planning as a result of self-configuring and self-organising networks, cognitive radio, multihop ad hoc communication and cross layer system design. A receiver experiences interference from transmitters within its accessibility radius, $R_{\text {ac }}$. Due to propagation path loss, a transmitter outside the accessibility region incurs only a negligible interference. Since the aim is to model a realistic interference limited environment, the receiver accessibility radius is taken to be much greater than the cell radius i.e $R_{\mathrm{ac}} \gg R_{\mathrm{c}}$. The dashed line in Fig. 1 represents the interference link between transmitter, Tx $y$, and receiver, $R x z$ while the solid line shows the desired link between transmitter Tx $x$ and receiver $\mathrm{Rx} z$ and vise versa. Throughout the derivation omni-directional antennas with unity gains are considered. The pdf is calculated assuming one interfering user. The results obtained can be extended to multiple interfering users by using laguerre polynomials to approximate the multiple integration resulting from the multiple interfering users. The analytical derivation of SIR for multiple interference is under study.

\section{Analytical derivation of the pdf of the sir}

In an interference limited environment, the received signal quality at a receiver is typically measured by means of achieved SIR, which is the ratio of the power of the wanted signal to the total residue power of the unwanted signals. let $P_{t}$ and $P_{r}$ denote the transmit and received power respectively. Let $G$ denote the path gain and $G_{y z}$ is the link gain between the interfering transmitter $y$ and the receiver $z$. For the purpose of clarity, unless otherwise stated, a single subscript $x, y$ or $z$ specifies the node, and a double subscript such as $x z$ specifies the link between node $x$ and node $z$. A node is any entity, mobile station(MS) or base station(BS) 


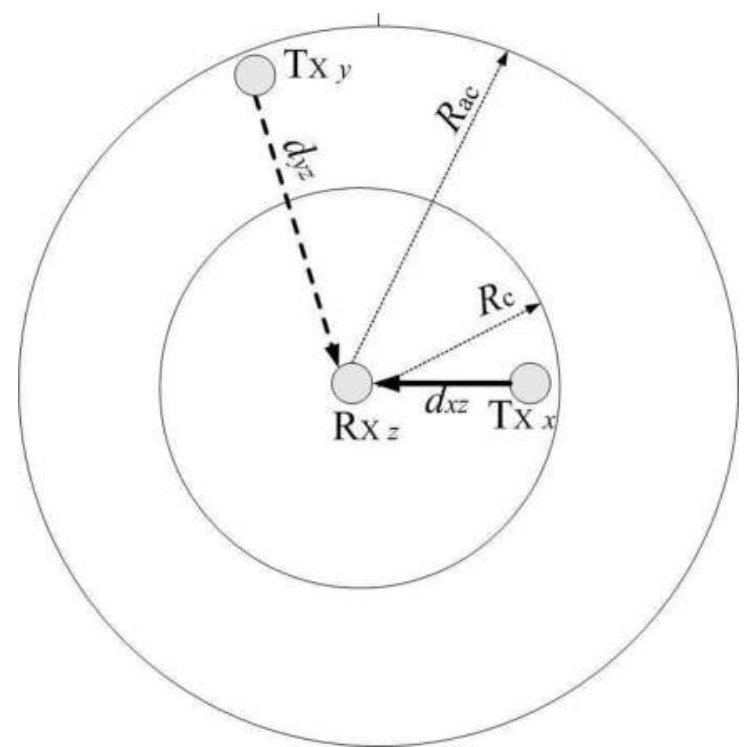

Fig. 1. Model to drive the pdf of the SIR from a single neighboring cell

that is capable of communicating. For a single interfering user $y$ depicted in Fig. 1:

$$
\operatorname{SIR}_{z}=\frac{P_{t x} G_{x z}}{P_{t y} G_{y z}}
$$

Assuming fixed and constant transmit powers, $P_{t x}=P_{t y}=$ const, (1) simplifies to:

$$
\begin{gathered}
\operatorname{SIR}_{z}=\frac{G_{x z}}{G_{y z}} \\
L=\frac{1}{G} \Rightarrow \operatorname{SIR}_{z}=\frac{L_{y z}}{L_{x z}}
\end{gathered}
$$

where $L_{x z}, L_{y z}$ are the path losses between transmitter Tx $x$ and receiver $\operatorname{Rx} z$ and Tx $y$ and $\mathrm{Rx} z$ respectively.

Like the gain parameter $G$, the loss parameter $L$ incorporates effects such as propagation loss, shadowing and multipath fading.

The generalized path loss model for the cross layer environment is given by:

$$
L=\underbrace{C\left(\frac{d}{d_{0}}\right)^{\gamma} \mathrm{e}^{(\beta \xi)}}_{\text {large scale path loss small scale path loss }} \cdot \underbrace{\frac{1}{|H(f)|^{2}}}_{\text {scif }}
$$

Where $C=\frac{\hat{C}}{\tilde{C}}$ is an environment specific constant, $\hat{C}$ the constant corresponding to the desired link while $\tilde{C}$ corresponds to the interference link. The distance $d_{0}$ is a constant and $d$ is a 
random variable, $\gamma$ is the path-loss exponent, $\xi$ is the random component due to shadowing, $\beta=\ln (10) / 10$ and $|H(f)|$ is a random variable modeling the channel envelop.

The commonly used path loss equation Rappaport (2001) only accounts for the large scale path loss with regular cell deployment scenarios, which is incomplete for studying self-organizing networks. The new path loss model proposed here takes into consideration the interaction of the large scale path loss as well as the small scale fading. This model is particularly important in studying the performance of self-organizing self-configuring networks.

For the interference scenario described in the system model, the path loss for the desired path and the path loss between the interfering transmitter $y$ and the receiver $z$ (interfering link) are:

$$
\begin{aligned}
& L_{x z}=\tilde{C} d_{x z}^{\gamma_{x z}} \mathrm{e}^{\left(\beta \xi_{x z}\right)} \frac{1}{\left|H_{x z}\right|^{2}} \\
& L_{y z}=\hat{C} d_{y z}^{\gamma_{y z}} \mathrm{e}^{\left(\beta \xi_{y z}\right)} \frac{1}{\left|H_{y z}\right|^{2}}
\end{aligned}
$$

where $L_{x z}$ is the path loss model for the desired link and $L_{y z}$ is the path loss model for the interfering link. $d_{y z}$ models the distance between the interference causing transmitter, $x$, and the victim receiver $y \cdot \gamma_{y z}$ and $\gamma_{x z}$ are the path loss exponents, $\xi_{x z}$ and $\xi_{y z}$ are Gaussian distributed random variables modeling the shadow fading with each zero mean and variances $v_{x z}^{2}$ and $v_{y z}^{2}$ respectively, and $\left|H_{x z}\right|$ and $\left|H_{y z}\right|$ are the channel envelope modeling the channel fading. For the purpose of clarity, the time and frequency dependencies are not shown. The channel envelope is assumed to follow the Nakagami- $m$ distribution. Nakagami distribution is a general statistical model which encompasses Rayleigh distribution as a special case, when the fading parameter $m=1$, and also approximates the Rician distribution very well. In addition, Nakagami-m distribution will also provide the flexibility of choosing different distributions for the desired link and interfering link, such as the Rayleigh for the channel envelope of the desired link, and Rician for the interfering link, or vice versa.

Using equations (3) and (5), the SIR can be given as:

$$
\mathrm{SIR}=\frac{C d_{y z}^{\gamma_{y z}} \mathrm{e}^{\left(\beta \xi_{y z}\right)}\left|H_{x z}\right|^{2}}{d_{x z}^{\gamma_{x z}} \mathbf{e}^{\left(\beta \xi_{x z}\right)}\left|H_{y z}\right|^{2}}
$$

From (7), the SIR has six random variable components, $\Phi_{x z}=d_{x z}^{\gamma_{x z}}, \Phi_{y z}=d_{y z}^{\gamma_{y z}}, \Lambda_{x z}=\mathrm{e}^{\left(\beta \xi_{x z}\right)}$, $\Lambda_{y z}=\mathrm{e}^{\left(\beta \xi_{y z}\right)},\left|H_{x z}\right|^{2}$ and $\left|H_{y z}\right|^{2}$. In order to analytically derive the pdf of the SIR, the pdf of the individual components and also their ratios and products need to be determined first.

The following two formulas provide the basic framework for the analysis and will be used throughout the derivation. Given two independent random variables $X$ and $Y$ the pdf of their product $f_{Z}(z)$ where $Z=X Y$ is

$$
f_{Z}(z)=\int f_{X}(z / x) f_{Y}(x)(1 /|x|) d x
$$

Given two independent random variables $Y$ and $X$ the pdf of their ratio $f_{Z}(z)$ where $Z=\frac{Y}{X}$ is

$$
f_{Z}(z)=\int f_{X}(x) f_{Y}(z x)|x| d x
$$




\subsection{Pdf of the ratio of the propagation loss}

It is assumed that the distance between the interfering transmitter and the receiver, $d_{y z}$, is uniformly distributed up to a maximum distance of $R_{\mathrm{ac}}$, and that the distance between an interfering transmitter and intended receiver, $d_{x z}$, is uniformly distributed up to a maximum distance of $R_{c}$. Therefore $\Phi_{x z}$ and $\Phi_{y z}$ are both functions of random variables, and their pdfs can be derived using the following random variable transformation Papoulis (1991).

$$
p(\theta)=\left.\frac{p(\delta)}{\left|\frac{d(\theta)}{d(\delta)}\right|}\right|_{\delta=\mathrm{F}^{-1}(\theta)}
$$

Where $\theta$ and $\delta$ are random variables with pdfs $p(\theta)$ and $p(\delta)$ respectivly, and where $\theta$ is a function of $\mathrm{F}(\mathrm{ffi}), d(\theta)$ and $d(\delta)$ are the first derivatives of $\theta$ and $\delta$ respectively.

The mathematical representation of the pdfs of $d_{x z}$ and $d_{y z}$ are

$$
\begin{aligned}
& f_{D_{x z}}\left(d_{x z}\right)=\frac{2 d_{x z}}{R_{\mathrm{c}}^{2}} \quad 0<d_{x z} \leq R_{\mathrm{c}} \\
& f_{D_{y z}}\left(d_{y z}\right)=\frac{2 d_{y z}}{R_{\mathrm{ac}}^{2}} \quad 0<d_{y z} \leq R_{\mathrm{ac}}
\end{aligned}
$$

Let $f_{\Phi_{x z}}\left(\phi_{x z}\right)$ and $f_{\Phi_{y z}}\left(\phi_{y z}\right)$ denote the pdfs of $\Phi_{x z}$ and $\Phi_{y z}$. Then employing the transformation (10), $f_{\Phi_{x z}}\left(\phi_{x z}\right)$ and $f_{\Phi_{y z}}\left(\phi_{y z}\right)$ are derived as

$$
\begin{aligned}
& f_{\Phi_{x z}}\left(\phi_{x z}\right)=\frac{2 \phi_{x z}^{2 / \gamma_{x z}-1}}{R_{\mathrm{c}}^{2} \gamma_{x z}} \quad 0<\phi_{x z} \leq R_{\mathrm{c}}^{\gamma_{x z}} \\
& f_{\Phi_{y z}}\left(\phi_{y z}\right)=\frac{2 \phi_{y z}^{2 / \gamma_{y z}-1}}{R_{\mathrm{ac}}^{2} \gamma_{y z}} \quad 0<\phi_{y z} \leq R_{\mathrm{ac}}^{\gamma_{y z}}
\end{aligned}
$$

Using (9), the pdf of the ratio of the propagation loss, $\Phi=\frac{\Phi_{y z}}{\Phi_{x z}}$, is found to be,

$$
f_{\Phi}(\phi)=\left\{\begin{aligned}
\mathrm{Y} \phi^{2 / \gamma_{y z}-1} & \text { for } 0<\phi \leq \varsigma \\
\tilde{Y} \phi^{-2 / \gamma_{x z}-1} & \text { for } \varsigma<\phi<\infty
\end{aligned}\right.
$$

where $\varsigma=\frac{R_{\mathrm{ac}}^{\gamma y z}}{R_{\mathrm{c}}^{\gamma(x z}}, \mathrm{Y}=\frac{2 R_{\mathrm{c}}^{2 \frac{\gamma_{x z}}{\mathrm{\gamma} y z}}}{R_{\mathrm{ac}}^{2}\left(\gamma_{y z}+\gamma_{x z}\right)}$ and $\tilde{Y}=\frac{2 R_{\mathrm{ac}}^{2 \frac{\gamma_{y z}}{\gamma y z}}}{R_{\mathrm{c}}^{2}\left(\gamma_{y z}+\gamma_{x z}\right)}$

The next step to derive the pdf of the SIR is find the pdf of the ratio of the lognormal shadowing.

\subsection{Pdf of the ratio of the lognormal shadowing}

Given a normally distributed random variable $X$ with mean $\mu$ and variance $\sigma^{2}$, and a real constant $c$, the product $c X$ is known to follow a normal distribution with mean $c \mu$ and a variance $c^{2} \sigma^{2}$ and $\mathrm{e}^{X}$ has a log-normal distribution. Since $\xi_{x z}$ is normally distributed with mean $\mu$ and variance $\sigma^{2}, \Lambda_{x z}=\mathrm{e}^{\left(\beta \xi_{x z}\right)}$ is a lognormal distributed random variable with mean $\mu_{x z}$ and variance $v_{x z}^{2}=\beta^{2} \sigma_{x z}{ }^{2}$ expressed in terms of the normally distributed $\xi_{x z}$, while the mean and variance of $\Lambda_{y z}=\mathrm{e}^{\left(\beta \xi_{y z}\right)}$ are $\mu_{y z}$ and $v_{y z}^{2}=\beta^{2} \sigma_{y} z^{2}$ respectively. 


$$
\begin{gathered}
f_{\Lambda_{x z}}\left(\lambda_{x z}\right)=\frac{\mathrm{e}^{-1 / 2 \frac{\left(\ln \left(\lambda_{x z}\right)-\mu_{x z}\right)^{2}}{v_{x z}{ }^{2}}}}{\lambda_{x z} v_{x z} \sqrt{2 \pi}}, \quad 0 \leq \lambda_{x z}<\infty \\
f_{\Lambda_{y z}}\left(\lambda_{y z}\right)=\frac{\mathrm{e}^{-1 / 2 \frac{\left(\ln \left(\lambda_{y z}\right)-\mu y z\right.}{v_{y z}{ }^{2}}}}{\lambda_{y z} v_{y z} \sqrt{2 \pi}}, \quad 0 \leq \lambda_{y z}<\infty
\end{gathered}
$$

Since the ratio of two independent lognormal random variables is itself a lognormal distributed random variable. Therefore the pdf of $\Lambda=\frac{\Lambda_{y z}}{\Lambda_{x z}}$ is:

$$
f_{\Lambda}(\lambda)=\frac{\mathrm{e}^{-1 / 2 \frac{(\ln (\lambda)-\mu)^{2}}{\sigma^{2}}}}{\lambda \sigma \sqrt{2 \pi}}, \quad 0 \leq \lambda<\infty
$$

where

$$
\sigma=\beta \sqrt{v_{x z}+v_{y z}}, \quad \mu=0 ;
$$

The last components remaining from (7) are the random variables modeling the channel envelop and their ratios.

\subsection{Pdf of the ratio of the channel envelope}

In order to accommodate different channel fading distributions, Nakagami- $m$ distribution was used to model the channel envelope. Nakagami- $m$ distribution is the most general of all distribution known until now Nakagami (1960).

The Nakagami- $m$ pdf is given by:

$$
\begin{aligned}
& f_{\left|H_{x z}\right|}\left(h_{x z}\right)=\frac{2}{\Gamma\left(m_{x z}\right)}\left(\frac{m_{x z}}{\Omega_{x z}}\right)^{m_{x z}} h_{x z} 2_{x z}-1 \mathrm{e}^{-\frac{m_{x} h_{x z} z^{2}}{\Omega_{x z}}}, \quad 0 \leq h_{x z}<\infty \\
& f_{\left|H_{y z}\right|}\left(h_{y z}\right)=\frac{2}{\Gamma\left(m_{y z}\right)}\left(\frac{m_{y z}}{\Omega_{y z}}\right)^{m_{y z}} h_{y z} 2 m_{y z}-1 \mathrm{e}^{-\frac{m_{y z} h_{y z}{ }^{2}}{\Omega_{y z}}}, \quad 0 \leq h_{y z}<\infty
\end{aligned}
$$

where $m \geq 1 / 2$ represents the fading figure, $\Omega=E\left(x^{2}\right)$ is the average received power and $\Gamma($.$) is the gamma function given as$

$$
\Gamma(m)=\int_{0}^{\infty} x^{m-1} \mathrm{e}^{-x} d x .
$$

The pdfs of the received instantaneous power, $H_{x z}=\left|H_{x z}\right|^{2}$ are modeled by a gamma distribution. For the desired user the pdf of the receive signal power, $f_{H_{x z}}\left(h_{x z}\right)$, is given as

$$
f_{H_{x z}}\left(h_{x z}\right)=\frac{h_{x z} m_{x z}-1}{\Gamma\left(m_{x z}\right)}\left(\frac{m_{x z}}{\Omega_{x z}}\right)^{m_{x z}} \mathrm{e}^{-\frac{m_{x z} h_{x z}}{\Omega_{x z}}}, \quad 0 \leq h_{x z}<\infty
$$

and for the interfering user the PDF, $f_{\left|H_{y z}\right|}\left(h_{y z}\right)$, is

$$
f_{H_{y z}}\left(h_{y z}\right)=\frac{h_{y z}^{m_{y z}-1}}{\Gamma\left(m_{y z}\right)}\left(\frac{m_{y z}}{\Omega_{y z}}\right)^{m_{y z}} \mathrm{e}^{-\frac{m_{y z} h_{y z}}{\Omega_{y z}}}, \quad 0 \leq h_{y z}<\infty
$$

Using (8) and (9) the pdf of the ratio of gamma distribution, $\Psi=\frac{H_{x z}}{H_{y z}}$ is: 


$$
f_{\Psi}(\psi)=M \frac{\psi^{m_{x z}-1}}{\left(\frac{m_{y z}}{\Omega_{y z}}+\frac{m_{x z}}{\Omega_{x z}} \psi\right)^{\left(m_{y z}+m_{x z}\right)}} \quad 0 \leq \psi<\infty
$$

where

$$
M=\frac{\Gamma\left(m_{y z}\right) \Gamma\left(m_{x z}\right)}{\Gamma\left(m_{y z}+m_{x z}\right)}\left(\frac{m_{y z}}{\Omega_{y z}}\right)^{m_{y z}}\left(\frac{m_{x z}}{\Omega_{x z}}\right)^{m_{x z}} .
$$

Using the beta function, also called the Euler integral of the first kind, $M$ can be re-written as

$$
M=\frac{\left(\frac{m_{y z}}{\Omega_{y z}}\right)^{m_{y z}}\left(\frac{m_{x z}}{\Omega_{x z}}\right)^{m_{x z}}}{\mathrm{~B}\left(m_{x z}, m_{y z}\right)}
$$

where

$$
\mathrm{B}\left(m_{x z}, m_{y z}\right)=\int_{0}^{1} t^{m_{x z}-1}(1-t)^{m_{y z}-1} d t=\frac{\Gamma\left(m_{y z}+m_{x z}\right)}{\Gamma\left(m_{y z}\right) \Gamma\left(m_{x z}\right)} .
$$

The final step in the derivation of the pdf of the SIR is deriving the product of the above obtained pdfs.

\subsection{Pdf of the SIR}

As shown in (7) the pdf of the SIR is the product of the three individual random variables, $\Phi$, $\Lambda$ and $\Psi$. Using the equations presented so far, the final pdf of the SIR is presented in (26)

$$
\begin{aligned}
& f_{\mathrm{SIR}}(\zeta)=M \zeta^{2 m_{x z}-1} \times \\
& \int_{0}^{\infty} \frac{A_{1} \chi^{q_{1}}\left(\operatorname{erf}\left(\frac{\frac{2}{\gamma y z} \sigma^{2}+\ln \left(\frac{\chi}{\left(\frac{R_{a c}^{\gamma y z}}{R_{c}^{\gamma x z}}\right)}\right)}{\sqrt{2} \sigma}\right)+B_{1} \chi^{q_{2}}\left(-1-\operatorname{erf}\left(\frac{\frac{-2}{\gamma x z} \sigma^{2}+\ln \left(\frac{\chi}{\left(\frac{R_{a c}^{\gamma y z}}{R_{c}^{\gamma \gamma z}}\right)}\right)}{\sqrt{2} \sigma}\right)\right)\right.}{\left(\frac{m_{y z}}{\Omega_{x z}}+\frac{m_{x z}}{\Omega_{y z}}\left(\frac{\zeta}{\chi}\right)^{2}\right)^{\left(m_{y z}+m_{x z}\right)}} d \chi
\end{aligned}
$$

where $q_{1}=\frac{2}{\gamma_{y z}}-2 m_{y z}-1, q_{2}=\frac{-2}{\gamma_{x z}}-2 m_{y z}-1$,

$$
A_{1}=\frac{-\frac{2 R_{\mathrm{c}}^{2 \frac{\gamma x z}{\gamma y z}}}{R_{\mathrm{ac}}^{2}\left(\gamma_{y z}+\gamma_{x z}\right)}}{2} \mathrm{e}^{\left(\frac{2}{\gamma_{y z}^{2}}\right) \sigma^{2}}
$$

and

$$
B_{1}=\frac{-\frac{2 R_{\mathrm{ac}}^{2 \frac{\gamma y z}{y z}}}{R_{\mathrm{c}}^{2}\left(\gamma_{y z}+\gamma_{x z}\right)}}{2} \mathrm{e}^{\left(\frac{2}{\gamma_{x z}^{2}}\right) \sigma^{2}}
$$

The final equation does not have a closed form solution but it is possible to solve the integration using numerical methods. 


\section{Signal to Interference and Noise Ratio}

In case of an environment that is is not interference limited, the SINR (signal to interference and noise ratio) is required to fully describe the communication channel. SINR can easily be found by modifying the SIR equation given in (1):

$$
\operatorname{SINR}_{z}=\frac{G_{x z}}{G_{y z}+N}
$$

where $N$ is the random variable modeling the Gaussian noise with mean $m_{N}=0$ and a standard deviation of $\sigma_{N}$. By applying the generalized path loss equation in (4), SINR at the receiver $\operatorname{Rx} z$ is given by:

$$
\operatorname{SINR}_{z}=\frac{\frac{\left|H_{x z}\right|^{2}}{d_{x z}^{\gamma x z} \mathrm{e}^{(\beta \xi \xi x z)}}}{\frac{\left|H_{y z}\right|^{2}}{d_{y z}^{\gamma y z} \mathrm{e}^{(\beta \xi \xi y z)}}+N}
$$

where the pdfs of the individual random variables are given in the previous section. let $\Theta_{x z}=$ $\Phi_{x z} \Lambda_{x z}=d_{x z} \gamma_{x z} \mathrm{e}^{\left(\beta \xi_{x z}\right)}$ which are derived in the previous section. The pdf of $\Theta_{x z}, f_{\Theta_{x z}}\left(\theta_{x z}\right)$, is given as:

$$
\begin{gathered}
f_{\Theta}\left(\theta_{x z}\right)=\int f_{\Phi}\left(\theta_{x z} / \lambda_{x z}\right) f_{\Lambda_{x z}}\left(\lambda_{x z}\right)\left(1 /\left|\lambda_{x z}\right|\right) d \lambda_{x z} \\
f_{\Theta}\left(\theta_{x z}\right)=\int_{\frac{\theta_{x z}}{R_{c}^{2}}}^{\infty} \frac{2\left(\frac{\theta_{x z}}{\lambda_{x z}}\right)^{2 / \gamma_{x z}-1}}{R_{c}^{2} \gamma_{x z}} \frac{\mathrm{e}^{-1 / 2 \frac{\beta\left(\ln \left(\lambda_{x z}\right)-\mu\right)^{2}}{v_{x z}}}}{\lambda_{x z} v_{x z} \sqrt{2 \pi}} \frac{1}{\lambda_{x z}} d \lambda_{x z} \\
f_{\Theta}\left(\theta_{x z}\right)=D\left(1-\frac{\operatorname{erf}\left(2 v_{x z}{ }^{2}-\gamma_{x z} m_{x z}+\gamma_{x z} \log \frac{\theta_{x z}}{R_{c}^{\gamma x z}}\right)}{\sqrt{(2) \gamma_{x z} v_{x z}}}\right)
\end{gathered}
$$

where $D=\frac{\mathrm{e}^{\frac{2 v x z^{2}-2 \gamma x z m x z}{\gamma x z^{2}}}}{R_{\mathrm{c}}^{2} \gamma_{x z}} \theta_{x z^{\frac{2}{\gamma x z-1}}}$

The next step in the derivation is to find the pdf of the path loss of the desired link by utilizing (9) and (19). Let $S=\frac{H_{x z}}{d_{x z}^{\gamma x z} \mathrm{e}^{(\beta \zeta x z)}}$ be the random variable denoting the path loss of the desired link. The pdf of $S$ is given as:

$$
f_{S}(s)=\mathrm{K} \int_{0}^{\infty} h_{x z} 2 m_{x z} \mathrm{e}^{-\frac{m_{x z} h_{x z}{ }^{2}}{\Omega x z}}\left(1-\frac{\operatorname{erf}\left(2 v_{x z}{ }^{2}-\gamma_{x z} m_{x z}+\gamma_{x z} \log \frac{s h_{x z}}{R_{c}^{\gamma / z z}}\right)}{\sqrt{(2) \gamma_{x z} v_{x z}}}\right) d h_{x z}
$$

where $\mathrm{K}=\frac{2}{\Gamma\left(m_{x z}\right)} \frac{m_{x z}{ }^{2}}{\Omega_{x z}} D$

The pdf of the path loss of the interference path denoted by the random variable $I=\frac{\left|H_{y z}\right|}{d_{y z}^{\gamma y z} \mathrm{e}^{\left(\beta \xi_{y z}\right)}}$ is give as:

$$
f_{I}(i)=\hat{\mathrm{K}} \int_{0}^{\infty} h_{y z}{ }^{2 m_{y z}} \mathrm{e}^{-\frac{m_{y z} h_{y z}{ }^{2}}{\Omega y z}}\left(1-\frac{\operatorname{erf}\left(2 v_{y z}{ }^{2}-\gamma_{y z} m_{y z}+\gamma_{y z} \log \frac{i h_{y z}}{R_{\mathrm{rv}} \gamma / 2}\right)}{\sqrt{(2)} \gamma_{y z} v_{y z}}\right) d h_{y z}
$$

where $\hat{\mathrm{K}}=\frac{2}{\Gamma\left(m_{y z}\right)} \frac{m_{y z}{ }^{2}}{\Omega_{y z}} D$ 
In order to find the pdf of the interference plus noise, $I+N$, it is assumed that interference is independent of noise. The pdf of the sum of two independent random variables $U$ and $V$, each of which has a probability density function, is the convolution of their individual density functions:

$$
f_{U+V}(z)=\int f_{U}(z-x) f_{V}(x) d x
$$

therefore the pdf of $I+N, f_{I+N}(z)$ is given by:

$$
f_{I+N}(z)=\int_{0}^{\infty} f_{I}(z-x) f_{N}(x) d x
$$

where $f_{N}(x)=\frac{\mathrm{e}^{-\frac{1}{2}\left(\frac{\mathrm{x}}{\mathrm{Q}_{N}}\right)^{2}}}{\sqrt{2 \pi} \sigma_{N}}$. Utilizing (9), the pdf of the SINR is given by :

$$
f_{\mathrm{SINR}}(v)=\int_{0}^{\infty} f_{I+N}(z) f_{S}(v z) z d z
$$

For the special case where the noise approaches zero, the pdf of the noise is represented as delta function or also known as, a unit impulse function, around zero. Therefore (35) can be rewritten as:

$$
f_{I+N}(z)=\int_{0}^{\infty} f_{I}(z-x) f_{N}(x) d x=\int_{0}^{\infty} f_{I}(z-x) \delta(x) d x=f_{I}(z)
$$

Thus

$$
f_{\mathrm{SINR}}(v)=\int_{0}^{\infty} f_{I+N}(z) f_{S}(v z) z d z=\int_{0}^{\infty} f_{I}(z) f_{S}(v z) z d z
$$

by the definition given in (9), the $f_{\text {SINR }}(v)$ given in (38) is the pdf of the SIR $\frac{S}{T}$. Therefore, when the noise approaches to zero, the pdf of the SINR given in (36) reduces to the pdf of SIR given in (26).

This sub-section has presented the pdf of the SINR as an extension to the pdf of the SIR. To validate the analytically derived SINR pdf, it is important to show that the core derivation, SIR derivation, is valid. The next sub-section validates the derivation through comparative numerical simulations of the SIR. The results presented were obtained using the adaptive Simpson quadrature numerical integration of the SIR.

\section{Results and discussion}

Monte Carlo simulations are carried out in order to validate the analytically derived pdf results. Fig. 2 and 3 show plots of the pdf of the SIR $f_{\text {SIR }}(\zeta)$ for different scenarios. The results presented in Figs. 2 - 4 show that the analytical pdf is in good agreement with the Monte Carlo simulation. The parameters used for the shadow fading, channel standard deviation and path loss exponents reflect a realistic deployment scenario for users moving at a speed of 25 to $40 \mathrm{~km} / \mathrm{h}$ Eltahir (2007). All simulations assume a channel envelope with a Nakagami$\mathrm{m}$ distribution with different $\mathrm{m}$ parameter, which corresponds to different fading scenario. These parameters are summarized in Tables 1- 3.

Fig. 2 depicts three different plots depending on the $\frac{R_{\mathrm{ac}}}{R_{c}}$. As the cell radius $R_{\mathrm{C}}$ increases there is a significant cell overlap leading to high mean value of interference which in turn leads to lower SIR mean value. Therefore, as the ratio of the cell radius to the accessibility radius approaches to one, the pdf is skewed towards smaller SIR. These plots show that the node with the lowest cell radius, $R_{\mathrm{c}}=100 \mathrm{~m}$, has the highest SIR mean. 
Fig. 3 shows the effect of different environments on the pdf of the SIR. The figure presents plots from an ad hoc free space outdoor deployment with line of sight scenario on the desired link, $\gamma=2$ and $m=3$, to the most severe non-line-of-sight scenario of obstructed indoor (in building) environment, $\gamma=4$ and $m=0.5$. The radius of the cell, $R_{\mathrm{c}}$, has been set to 100 $\mathrm{m}$, which is considered a good configuration example for ad hoc networks. The accessability radius, $R_{\mathrm{ac}}$ is assumed to be $500 \mathrm{~m}$. The results illustrate that the node with the best line-ofsight (LOS) link, $\gamma_{x z}=2$ and $\gamma_{y z}=4$, has the highes mean SIR value and the biggest variance or spread. While the node with the most obstructed inbuilding environment, exhibits the lowest mean and the smallest variance or spread of all. These can be attributed to the higher interference contribution of interfering node in NLOS link than those in LOS condition.

Fig. 4 present the cumulative density function of the SIR. The simulation parameters are summarized in table 3. From Fig. 4 it can be observed that for a target SIR of $25 \mathrm{~dB}$, being a reasonable assumption for 64-QAM modulation, the probability that the SIR exceeds the target SIR in the most severe non-line-of-sight scenario of obstructed indoor (in building) environment is about $10 \%$ resulting in a high outage probability enforcing the use of lower order modulation schemes. On the other hand, for the link with best LOS condition of outdoor free space environment the the probability that the SIR exceeds the target SIR is $85 \%$ allowing the use of higher order modulation. Therefore from the results in Fig. 4, it can be deducted that the analytical work presented in this chapter can be used in determining the boundaries for varying the modulation order. A similar work of determining the boundaries for adaptive modulation was presented by Goldsmith et al. Alouini \& Goldsmith (2000) assuming Nakagami distribution thus ignoring the shadowing effect, the pdf presented here can be used to extend the results presented in Alouini \& Goldsmith (2000).

\section{Conclusion}

The main contribution of this chapter is the derivation of the pdf of the SIR for cross layer design without recourse to Monte Carlo simulations. The derivation was carried out using a generalized path loss model that accounts for both large and small scale path loss. The use of Nakagami- $m$ distribution for the fading channel gives the flexibility to use Rayleigh or different channel fading models for the desired and interfering links. The results obtained show excellent agreement with the Monte Carlo based results. The SIR derivation was in turn used to derive the pdf of the SINR. The SINR derivation is important in non-interference limited environment. These derivations can be further used in applications where the knowledge of SIR is necessary, such as link adaptation algorithms and cognitive radio design. The analytical derivation of the pdf from a single interferer in this chapter lays a solid foundation to calculate the statistics from multiple interferers. 


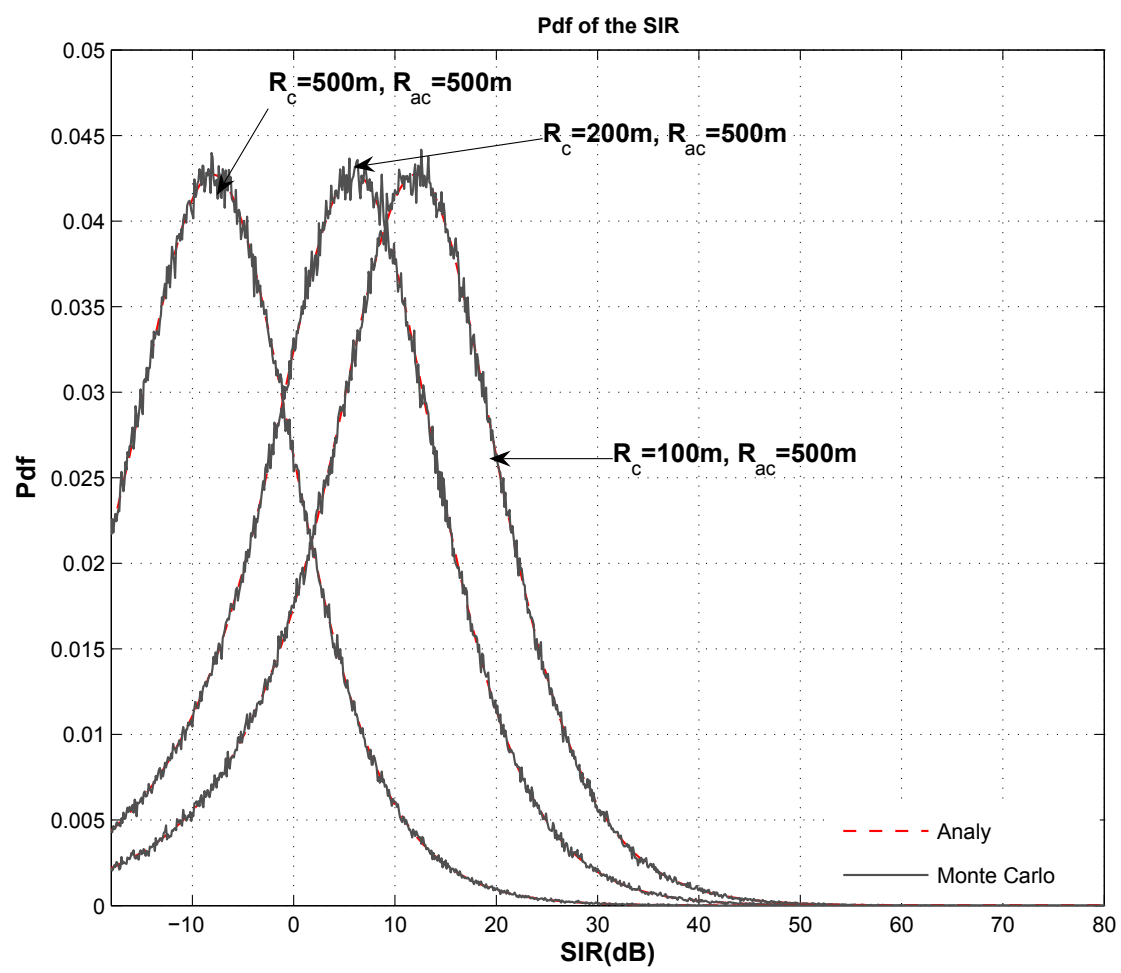

Fig. 2. Plots of the pdf of the SIR for different values of cell radius

\begin{tabular}{c|c}
\hline Parameter & Values \\
\hline$R_{\mathrm{c}}$ & $100 \mathrm{~m}$ \\
\hline$R_{\mathrm{ac}}$ & $500 \mathrm{~m}$ \\
\hline$v_{x z}$ & $6 \mathrm{~dB}$ \\
\hline$v_{y z}$ & $10 \mathrm{~dB}$ \\
\hline$\gamma_{x z}$ & 2 \\
\hline$\gamma_{y z}$ & 4 \\
\hline$m_{x z}$ & 5 \\
\hline$m_{y z}$ & 0.5 \\
\hline$\Omega_{x z}$ & $4 \mathrm{~dB}$ \\
\hline$\Omega_{y z}$ & $6 \mathrm{~dB}$ \\
\hline
\end{tabular}

Table 1. System parameters for Fig. 2(varying cell and accessability radius) 


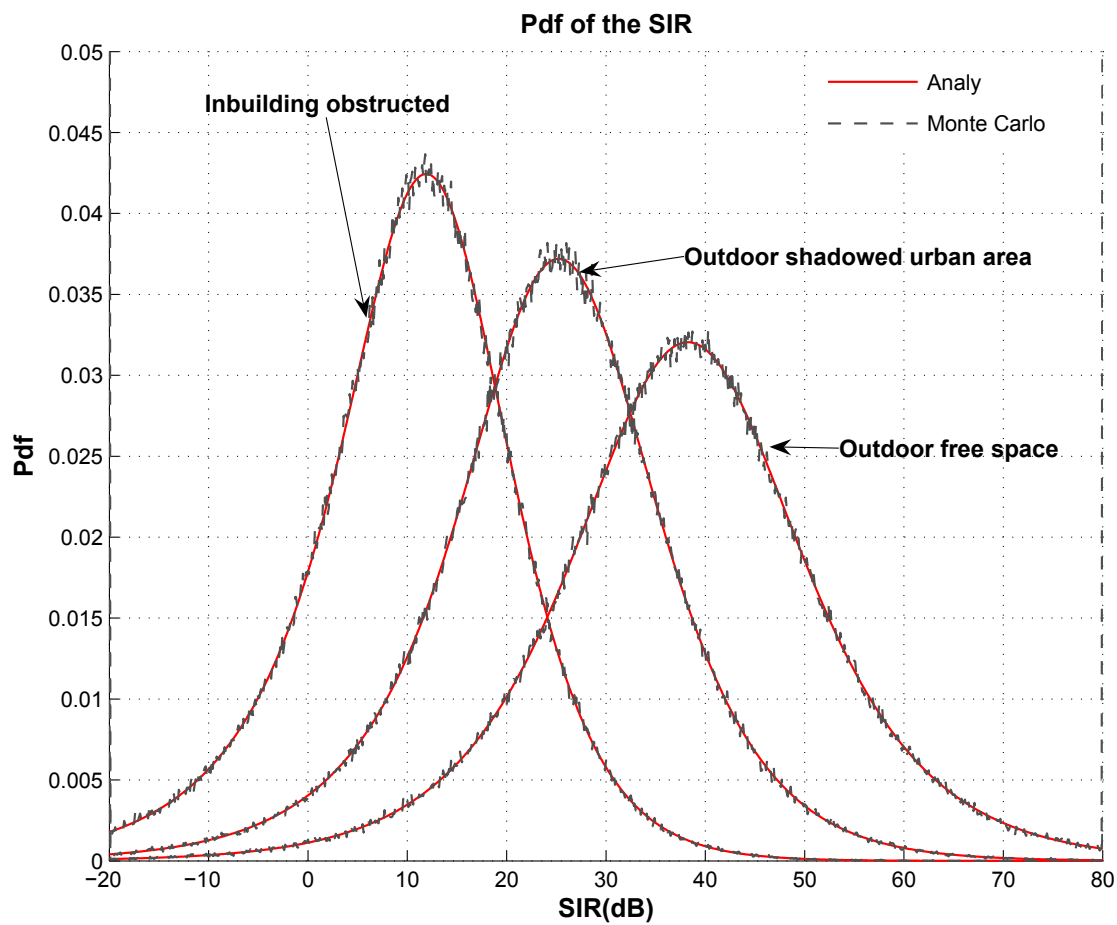

Fig. 3. Plots of the pdf of the SIR for different environments

\begin{tabular}{c|c|c|c}
\hline Parameter & Inbuilding obstructed & Outdoor shadowed urban & Outdoor free space \\
\hline$R_{\mathrm{c}}$ & $100 \mathrm{~m}$ & $100 \mathrm{~m}$ & $100 \mathrm{~m}$ \\
\hline$R_{\mathrm{ac}}$ & $500 \mathrm{~m}$ & $500 \mathrm{~m}$ & $500 \mathrm{~m}$ \\
\hline$v_{x z}$ & $10 \mathrm{~dB}$ & $8 \mathrm{~dB}$ & $10 \mathrm{~dB}$ \\
\hline$v_{y z}$ & $10 \mathrm{~dB}$ & $10 \mathrm{~dB}$ & $10 \mathrm{~dB}$ \\
\hline$\gamma_{x z}$ & 4 & 3 & 4 \\
\hline$\gamma_{y z}$ & 4 & 4 & 4 \\
\hline$m_{x z}$ & 3 & 1 & 0.5 \\
\hline$m_{y z}$ & 0.5 & 0.5 & 0.5 \\
\hline$\Omega_{x z}$ & $4 \mathrm{~dB}$ & $4 \mathrm{~dB}$ & $4 \mathrm{~dB}$ \\
\hline$\Omega_{y z}$ & $6 \mathrm{~dB}$ & $4 \mathrm{~dB}$ & $6 \mathrm{~dB}$ \\
\hline
\end{tabular}

Table 2. System parameters for Fig. 3 


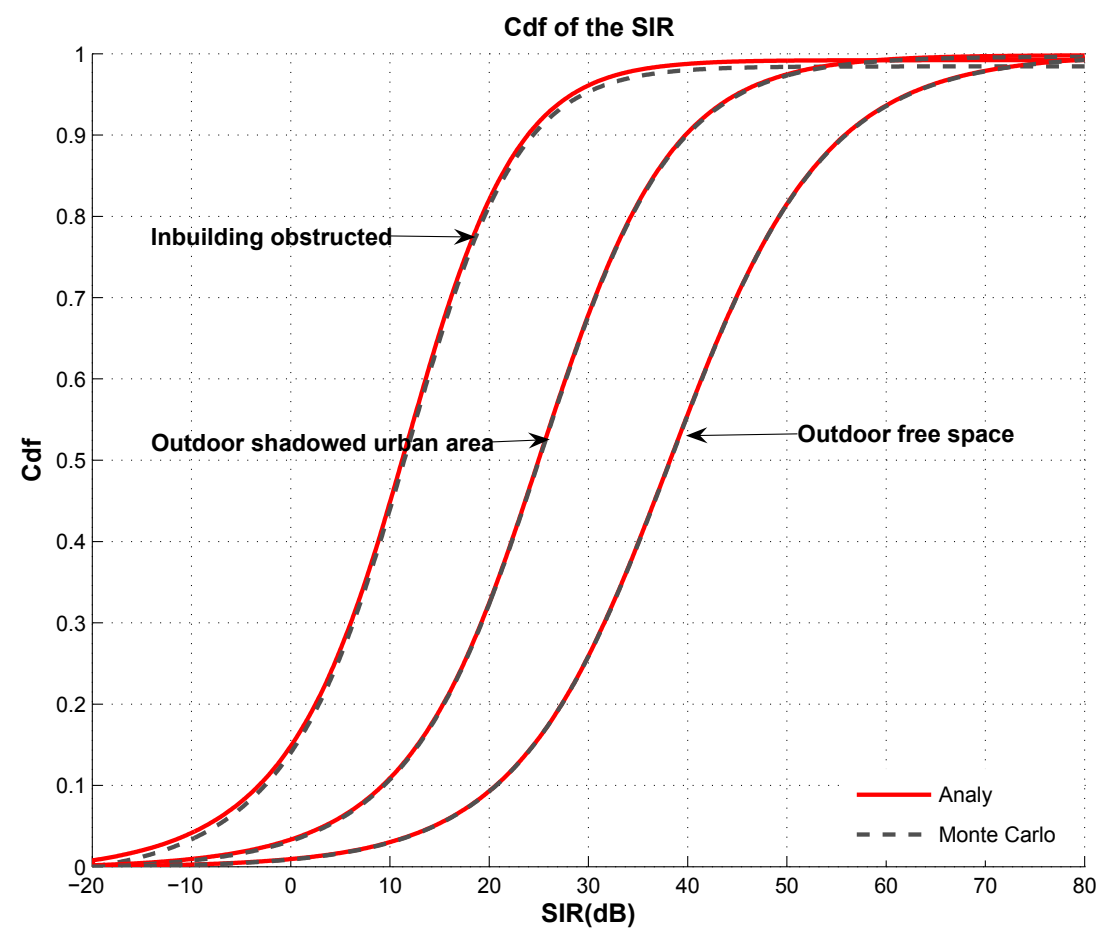

Fig. 4. Plots of the pdf of the SIR for different environments

\begin{tabular}{c|c|c|c}
\hline Parameter & Inbuilding obstructed & Outdoor shadowed urban & Outdoor free space \\
\hline$R_{\mathrm{c}}$ & $100 \mathrm{~m}$ & $100 \mathrm{~m}$ & $100 \mathrm{~m}$ \\
\hline$R_{\mathrm{ac}}$ & $500 \mathrm{~m}$ & $500 \mathrm{~m}$ & $500 \mathrm{~m}$ \\
\hline$v_{x z}$ & $10 \mathrm{~dB}$ & $8 \mathrm{~dB}$ & $10 \mathrm{~dB}$ \\
\hline$v_{y z}$ & $10 \mathrm{~dB}$ & $10 \mathrm{~dB}$ & $10 \mathrm{~dB}$ \\
\hline$\gamma_{x z}$ & 4 & 3 & 4 \\
\hline$\gamma_{y z}$ & 4 & 4 & 4 \\
\hline$m_{x z}$ & 3 & 1 & 0.5 \\
\hline$m_{y z}$ & 0.5 & 0.5 & 0.5 \\
\hline$\Omega_{x z}$ & $4 \mathrm{~dB}$ & $4 \mathrm{~dB}$ & $6 \mathrm{~dB}$ \\
\hline$\Omega_{y z}$ & $6 \mathrm{~dB}$ & $4 \mathrm{~dB}$ & $6 \mathrm{~dB}$ \\
\hline
\end{tabular}

Table 3. System parameters for Fig. 4 


\section{References}

Ahn, G.-S., Campbell, A., Veres, A. \& Sun, L.-H. (2002). Support Service Differentiation for Real-Time and Best-Effort Traffic in Stateless Wireless Ad Hoc Networks (SWAN), IEEE Trans. Mobile Comput. 1(3): 192-207.

Alouini, M.-S. \& Goldsmith, A. (1997). Capacity of Nakagami Multipath Fading Channels, Proc. of the IEEE Vehicular Technology Conference(VTC), Vol. 1, Arizona, USA, pp. 358362.

Alouini, M.-S. \& Goldsmith, A. (2000). Adaptive Modulation over Nakagami Fading Channels, Kluwer Journal on Wireless Commun. 13(1-2): 119-143.

Eltahir, I. (2007). The Impact of Different Radio Propagation Models for Mobile Ad hoc NETworks (MANET) in Urban Area Environment, Proc. of the International Conference on Wireless Broadband and Ultra Wideband Communications (AusWireless), Sydney, Australia, pp. 30-30.

Nakagami, M. (1960). The m-distribution: A General Formula of Intensity Distribution, Statistical Methods of Radio Wave Propagation, W. C. Hoffman, Ed., New York, Pergamon pp. 3-36.

Papoulis, A. (1991). Probability, Random Variables, and Stochastic Processes, 3 edn, McGraw-Hill.

Rappaport, T. S. (2001). Wireless Communications: Principles and Practice, 2 edn, Prentice Hall PTR.

Tellambura, C. (1999). Cochannel Interference Computation for Arbitrary Nakagami Fading, IEEE Trans. Veh. Technol. 48(2): 487-489.

Xia, X. \& Liang, Q. (2005). Bottom-up Cross-layer Optimization for Mobile ad hoc Networks, Proc. of the IEEE Military Communications Conference (MILCOM), Vol. 4, Atlantic City, USA, pp. 2624-2630.

Yeh, E. \& Cohen, A. (2003). A Fundamental Cross-layer Approach to Uplink Resource Allocation, Proc. of the IEEE Military Communications Conference (MILCOM), Vol. 1, Monterey, CA, pp. 699-704.

Yun, Z. \& Iskander, M. (2004). Progress in Modeling Challenging Propagation Environments, Proc. of the IEEE Antennas and Propagation Society International Symposium, Vol. 4, California, USA, pp. 3637-3640.

Zhang, Q. (1996). Outage Probability in Cellular Mobile Radio Due to Nakagami Signal and Interferers With Arbitrary Parameters, IEEE Trans. Veh. Technol. 45(2): 364-372.

Zorzi, M. (1997). On the Analytical Computation of the Interference Statistics with Applications to the Performance Evaluation of Mobile Radio Systems, IEEE Trans. Commun. 45(1): 103-109. 


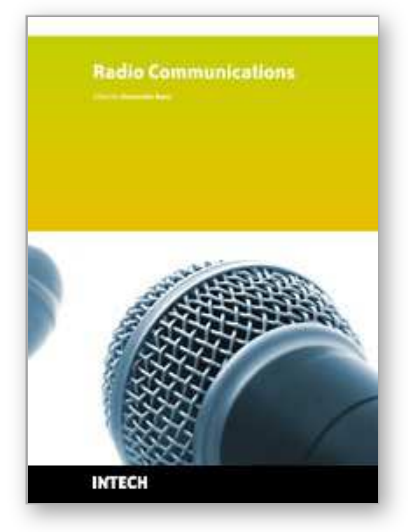

\author{
Radio Communications \\ Edited by Alessandro Bazzi
}

ISBN 978-953-307-091-9

Hard cover, 712 pages

Publisher InTech

Published online 01, April, 2010

Published in print edition April, 2010

In the last decades the restless evolution of information and communication technologies (ICT) brought to a deep transformation of our habits. The growth of the Internet and the advances in hardware and software implementations modified our way to communicate and to share information. In this book, an overview of the major issues faced today by researchers in the field of radio communications is given through 35 high quality chapters written by specialists working in universities and research centers all over the world. Various aspects will be deeply discussed: channel modeling, beamforming, multiple antennas, cooperative networks, opportunistic scheduling, advanced admission control, handover management, systems performance assessment, routing issues in mobility conditions, localization, web security. Advanced techniques for the radio resource management will be discussed both in single and multiple radio technologies; either in infrastructure, mesh or ad hoc networks.

\title{
How to reference
}

In order to correctly reference this scholarly work, feel free to copy and paste the following:

Abdurazak Mudesir and Harald Haas (2010). Analytical SIR for Cross Layer Channel Model, Radio Communications, Alessandro Bazzi (Ed.), ISBN: 978-953-307-091-9, InTech, Available from: http://www.intechopen.com/books/radio-communications/analytical-sir-for-cross-layer-channel-model

\section{INTECH}

open science | open minds

\author{
InTech Europe \\ University Campus STeP Ri \\ Slavka Krautzeka 83/A \\ 51000 Rijeka, Croatia \\ Phone: +385 (51) 770447 \\ Fax: +385 (51) 686166 \\ www.intechopen.com
}

\author{
InTech China \\ Unit 405, Office Block, Hotel Equatorial Shanghai \\ No.65, Yan An Road (West), Shanghai, 200040, China \\ 中国上海市延安西路65号上海国际贵都大饭店办公楼 405 单元 \\ Phone: +86-21-62489820 \\ Fax: +86-21-62489821
}


(C) 2010 The Author(s). Licensee IntechOpen. This chapter is distributed under the terms of the Creative Commons Attribution-NonCommercialShareAlike-3.0 License, which permits use, distribution and reproduction for non-commercial purposes, provided the original is properly cited and derivative works building on this content are distributed under the same license. 